\title{
Antenna Array Diagnosis for Millimeter-Wave MIMO Systems
}

\author{
Siqi Ma, Wenqian Shen, Jianping An, Lajos Hanzo, Fellow, IEEE
}

\begin{abstract}
The densely packed antennas of millimeter-Wave (mmWave) MIMO systems are often blocked by the rain, snow, dust and even by fingers, which will change the channel's characteristics and degrades the system's performance. In order to solve this problem, we propose a cross-entropy inspired antenna array diagnosis detection (CE-AAD) technique by exploiting the correlations of adjacent antennas, when blockages occur at the transmitter. Then, we extend the proposed CE-AAD algorithm to the case, where blockages occur at transmitter and receiver simultaneously. Our simulation results show that the proposed CE-AAD algorithm outperforms its traditional counterparts.
\end{abstract}

Index Terms-Millimter-Wave MIMO, antenna array diagnosis, cross-entropy.

\section{INTRODUCTION}

Millimeter-Wave (MmWave) multiple-input multiple-output (MIMO) techniques have a high promise for next-generation wireless communication systems due to the abundance of bandwidth [1], [2]. The short wave lengths of mmWave frequencies allow a large number of antennas to be packed in a compact physical size for providing a high beamforming gain for compensating the heavy path loss of mmWave signals. Unfortunately, these densely packed antennas are subject to blockages by dust, snow, water drops or fingers [3]-[5]. These blockages will change the array's geometry, causing a change of the composite channels [6]. Therefore, it is necessary to diagnose the blocked/faulty antennas.

Hence several antenna array diagnosis (AAD) techniques have been proposed in [6]-[12]. P. Zhang et al. [6] proposed an atomic norm based algorithm for detecting the position of blocked antennas. Bucci et al. [7] studied both the partial blockage as well as complete blockage, and then proposed a modified genetic algorithm for calculating the characteristic parameters of blocked antennas. Bucci et al. [8] also proposed a method for analysing near-field data in order to detect the location of blocked antennas. However, these AAD methods proposed in [6]-[8] require a large number of measurements, which will substantially increase the time required for the diagnosis. To solve this problem, sophisticated AAD techniques based on compressive sensing (CS) were proposed in [9]-[12]. Specifically, the AAD problem was formulated as a sparse signal recovery problem by Migliore in [9], which

S. Ma, W. Shen, J. An are with the Department of Information and Electronics, Beijing Institute of Technology, Beijing 100081, China (E-mails: sqma@bit.edu.com; wshen@bit.edu.cn; an@bit.edu.cn). L. Hanzo is with the Department of Electronics and Computer Science, University of Southampton, Southampton SO17 1BJ, UK (e-mail: 1h@ecs.soton.ac.uk).

L. Hanzo would like to acknowledge the financial support of the Engineering and Physical Sciences Research Council projects EP/Noo4558/1, EP/PO34284/1, COALESCE, of the Royal Society's Global Challenges Research Fund Grant as well as of the European Research Council's Advanced Fellow Grant QuantCom. can be solved by CS algorithms. As further developments, a powerful Bayesian method [10] and an iterative reweighted least square (IRLS) method [11] was also proposed for solving the problem of AAD. However, the above algorithms do not exploit the correlations between the blocked antennas. By contrast, upon exploiting the correlations between blocked antennas, a group-blockage diagnosis technique was proposed by Eltayeb et al. [12]. However, this method cannot be used in the scenario where both the receive and transmit antennas are blocked at the same time.

Against this background, we propose a cross-entropyinspired AAD method (CE-AAD) for detecting the location of blocked antennas and for calculating the corresponding characteristic parameters of blocked antennas. Specifically, our proposed CE-AAD exploits the correlations among the blocked antennas, where the the number of measurements is reduced. Then, we extend the proposed CE-AAD method to the scenario, where blockages occur both at the transmitter and receiver. Finally, our simulations show that the proposed algorithm outperforms its traditional counterparts in terms of its normalized mean square error (NMSE) performance.

The rest of the paper is organized as follows. In Section II, we present the system model. In Section III, we propose a CE-AAD algorithm for calculating the corresponding characteristic parameters of blocked antennas. In Section IV, we extend the proposed CE-AAD method to the scenario, where blockages occur both at the transmitter and receiver. Our simulation results are provided in Section V. Finally, our conclusions are drawn in Section VI.

Notation: We use the following notation throughout the paper. We let a, a, A represent the scalar, vector, and matrix respectively. $\operatorname{vec}\{\cdot\}$ denotes the vectorization of a matrix. ivec $\{\cdot\}$ denotes the invectorization of a vector. $(\cdot)^{\mathrm{T}},(\cdot)^{\mathrm{H}}$, and $(\cdot)^{-1}$ denote the transpose, conjugate transpose, and inverse of a matrix, respectively. The operator $\circ$ and $\otimes$ represent the Hadamard-product and Kronecker product, respectively. $\mathbf{1}_{N \times M}$ denotes all 1 matrix of size $N \times M$.

\section{SySTEM MODEL}

In this section, we consider a uniform planar array (UPA) of antennas at the transmitter with $N_{x}$ equally spaced elements along the x-axis, and $N_{y}$ equally spaced elements along the $\mathrm{y}$-axis. Thus the total number of antennas at the transmitter is $N_{\mathrm{t}}=N_{x} \times N_{y}$. The receiver is equipped with a single antenna. At the $k$-th measurement $(k=1,2 \cdots K)$, the received signal $g_{k}$ can be described as

$$
g_{k}=\mathbf{f}_{k}^{\mathrm{T}} \cdot \operatorname{vec}(\mathbf{H}) s+n_{k},
$$


where $\mathbf{H} \in \mathbb{C}^{N_{x} \times N_{y}}$ is the channel matrix, $\mathbf{f}_{k} \in \mathbb{C}^{N_{t} \times 1}$ is the transmit precoding (TPC) vector at the $k$-th measurement, and $s$ is the transmitted symbol, while $n_{k} \sim C N\left(0, \delta^{2}\right)$ is the additive noise and $\delta^{2}$ is the noise power. Considering the classical multi-path channel model, $\mathbf{H}$ can be written as [13]

$$
\mathbf{H}=\sum_{\ell=1}^{L} \beta_{\ell} \cdot \mathbf{A}\left(\theta_{\ell}, \varphi_{\ell}\right)
$$

where $L$ is the number of propagation paths and $\beta_{\ell}$ is the complex gain of the $\ell$-th path. Furthermore, $\mathbf{A}\left(\theta_{\ell}, \varphi_{\ell}\right) \in$ $\mathbb{C}^{N_{x} \times N_{y}}$ is the antenna array response, where $\theta_{\ell}$ is the elevation angle-of-departure (AoD) and $\varphi_{\ell}$ is the azimuth AoD. The $(m, n)$-th element of $\mathbf{A}\left(\theta_{\ell}, \varphi_{\ell}\right)$ is given by $\frac{1}{\sqrt{N_{x} N_{y}}} e^{j(m-1) \frac{2 \pi d_{x}}{\lambda} \sin \theta_{\ell} \cos \varphi_{\ell}} e^{j(n-1) \frac{2 \pi d_{y}}{\lambda} \sin \theta_{\ell} \sin \varphi_{\ell}}$ [13], where $d_{x}$ and $d_{y}$ are the antenna spacing along the $\mathrm{x}$-axis and $\mathrm{y}$-axis, $\lambda$ is the wavelength.

When the antenna array is blocked, the received signal at the $k$-th measurement is given by

$$
r_{k}=\mathbf{f}_{k}^{\mathrm{T}} \cdot(\mathbf{b} \circ \operatorname{vec}(\mathbf{H})) s+n_{k},
$$

where $\mathbf{b} \in \mathbb{C}^{N_{\mathrm{t}} \times 1}$ and the $n$-th element of vector $\mathbf{b}$ can be defined as

$$
b_{n}=\left\{\begin{array}{cc}
a_{n} & \text { if the } n \text {-th antenna is blocked } \\
1 & \text { otherwise }
\end{array},\right.
$$

where $a_{n}=\tau_{n} e^{i \Psi_{n}}$ is the characteristic parameter of the blocked antenna $n$. Here $\tau_{n}$ is the absorption coefficient following the uniform distribution between 0 and 1 . If the $n$-th antenna is completely blocked, $\tau_{n}$ is equal to 0 . Otherwise, $\tau_{n}$ is a positive value less than $1 . \Psi_{n}$ denotes the blockage scattering coefficient following the uniform distribution between 0 and $2 \pi$ [14].

Without loss of generality, we let the training symbol $s$ be equal to 1 . using (3) - (1), we have

$$
y_{k}=r_{k}-g_{k}=\mathbf{f}_{k}^{\mathrm{T}} \underbrace{(\operatorname{vec}(\mathbf{H}) \circ \mathbf{b}-\operatorname{vec}(\mathbf{H}))}_{\mathbf{q}}+\widetilde{n}_{k},
$$

where $\mathbf{q}=\left[q_{1}, q_{2}, \cdots, q_{N_{\mathrm{t}}}\right]^{\mathrm{T}} \in \mathbb{C}^{N_{\mathrm{t}} \times 1}, \widetilde{n}_{k} \sim C N\left(0, \delta^{2}\right)$. Note that the true channel matrix $\mathbf{H}$ is assumed to be known at the receiver. After $K$ measurements, we obtain

$$
\mathbf{y}=\mathbf{F q}+\widetilde{\mathbf{n}},
$$

where $\mathbf{y}=\left[y_{1}, y_{2}, \cdots y_{K}\right]^{\mathrm{T}} \in \mathbb{C}^{K \times 1}$ and $\mathbf{F}=$ $\left[\mathbf{f}_{1}, \mathbf{f}_{2}, \cdots \mathbf{f}_{K}\right]^{\mathrm{T}} \in \mathbb{C}^{K \times N_{\mathrm{t}}}$. The TPC matrix $\mathbf{F}$ can be designed as a random matrix, which can be realized by 2-bit phase shifters, whose elements are randomly selected from $\{1+i, 1-i,-1-i,-1+i\}$ [12]. Since the number of blocked antennas is usually small, the vector $\mathbf{q}$ is a sparse vector. Thus, the AAD problem becomes a sparse signal recovery problem. Through the estimation of $\mathbf{q}$, which is denoted by $\hat{\mathbf{q}}$, the locations of blocked antennas can be recognized by finding the positions of non-zero elements of $\widehat{\mathbf{q}}$. Then, the characteristic parameters of blocked antennas $\tau_{n}$ and $\Psi_{n}$ can be readily obtained as $\tau_{n}=\left|\frac{\widehat{q}_{n}}{h_{n}}+1\right|, \Psi_{n}=\angle\left(\frac{\widehat{q}_{n}}{h_{n}}+1\right)$, where $n \in \mathcal{S}_{q}$, which is the index set of non-zero elements in the vector q. Furthermore, $\mathbf{h}=\operatorname{vec}(\mathbf{H})$ and $h_{n}$ is the $n$-th element of $\mathbf{h}$. While $\widehat{q}_{n}$ denotes the $n$-th element of $\widehat{\mathbf{q}}$.

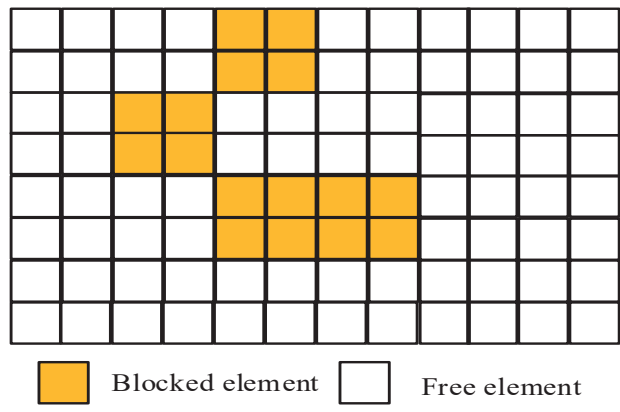

Fig. 1. An example of the block-sparse matrix $\mathbf{Q}$.

\section{Proposed CE-AAD Algorithm}

Let us now outline the CE-AAD algorithm by exploiting the correlations between the adjacent blocked antennas to detect the location of blocked antennas and calculate the corresponding characteristic parameters.

The short wave length of mmWave carriers allows a large number of antennas to be packed in a compact physical size. When dust, snow or water drops are attached to the antenna array at the transmitter, it is also very likely to affect the adjacent antennas, which results in the matrix $\mathbf{Q}=\operatorname{ivec}(\mathbf{q}) \in \mathbb{C}^{N_{x} \times N_{y}}$ becoming of block-sparse structure, as shown in Fig. 1. This situation has also been elaborated on in [12].

By exploiting the block-sparsity of matrix $\mathbf{Q}$, we propose a CE-AAD algorithm for recovering $Q$ using a low number of measurements. Specifically, we use the vector $\mathbf{d}$ of size $N_{\mathrm{t}} \times 1$ to denote the positions of non-zero elements of vector $\mathbf{q}$, where the elements of $\mathbf{d}$ are selected from $\{0,1\}$. That is to say, $q_{n}=0$ means $d_{n}=0$ and $q_{n} \neq 0$ means $d_{n}=1$, where $d_{n}$ is the $n$-th element of $\mathbf{d}$. Thus, we can rewrite (6) as

$$
\mathbf{y}=\mathbf{F}(\mathbf{q} \circ \mathbf{d})+\widetilde{\mathbf{n}} .
$$

Next, we propose the CE-AAD algorithm for estimating $\mathbf{d}$ and $\mathbf{q}$, as shown in Algorithm 1. Firstly, we initialize the algorithm by setting the probability matrix to $\mathbf{P}_{\mathrm{B}}^{0}=$ $\frac{1}{2} \times \mathbf{1}_{\left(N_{x} / N_{b_{x}}\right) \times\left(N_{y} / N_{b_{y}}\right)}$. We assume that the size of the rectangular blockages is fixed and can be described as $N_{b_{x}} \times N_{b_{y}}$. Thus, the matrix $\mathbf{Q}$ is divided into $\frac{N_{x} N_{y}}{N_{b_{x}} N_{b_{y}}}$ groups, where the elements of each group are either all non-zero or all zero.

In Step 1, when we generate the candidate matrix $\mathbf{Q}_{n_{c}}$, we consider the adjacent $N_{b_{x}} \times N_{b_{y}}$ elements of matrix $\mathbf{Q}_{n_{c}}$ as a group. Thus, we divide the matrix $\mathbf{Q}$ into $\left(N_{x} / N_{b_{x}}\right) \times$ $\left(N_{y} / N_{b_{y}}\right)$ blocks by exploiting the block-sparsity of $\mathbf{Q}$. We then define the probability matrix in the $i$-th iteration $\mathbf{P}_{\mathrm{B}}^{i} \in \mathbb{C}^{N_{x} / N_{b_{x}} \times N_{y} / N_{b_{y}}}$, where the $(m, n)$-th element of $\mathbf{P}_{\mathrm{B}}^{i}$ denotes the probability that $\mathbf{C}_{\mathrm{B}}^{n_{c}}(m, n)=1$. Here, $\mathbf{C}_{\mathrm{B}}^{n_{c}}(m, n)$ is the $(m, n)$-th element of $\mathbf{C}_{\mathrm{B}}^{n_{c}} \in\{0,1\}^{\left(N_{x} / N_{b_{x}}\right) \times\left(N_{y} / N_{b_{y}}\right)}$, indicating whether the $(m, n)$-th block of candidate matrix $\mathbf{Q}_{n_{c}}$ is a non-zero group. Then, we generate $N_{c}$ candidates $\left\{\mathbf{C}_{\mathrm{B}}^{n_{c}}\right\}_{n_{c}=1}^{N_{c}}$ based on the probability matrix $\mathbf{P}_{\mathrm{B}}^{i}$. Then, we vectorize $\mathbf{C}_{n_{c}}$ as $\mathbf{d}_{n_{c}}=\operatorname{vec}\left(\mathbf{C}_{n_{c}}\right) \in \mathbb{R}^{N_{\mathrm{t}} \times 1}$, where $\mathbf{C}_{n_{c}}=$ $\mathbf{C}_{\mathrm{B}}^{n_{c}} \otimes \mathbf{1}_{N_{b_{x}} \times N_{b_{y}}} \in \mathbb{R}^{N_{x} \times N_{y}}$. We also define the index set $\mathcal{D}_{n_{c}}$ for recording the indices of non-zero elements in vector $\mathbf{d}_{n_{c}}$. In Step 2, we use the least squares (LS) [15] algorithm 


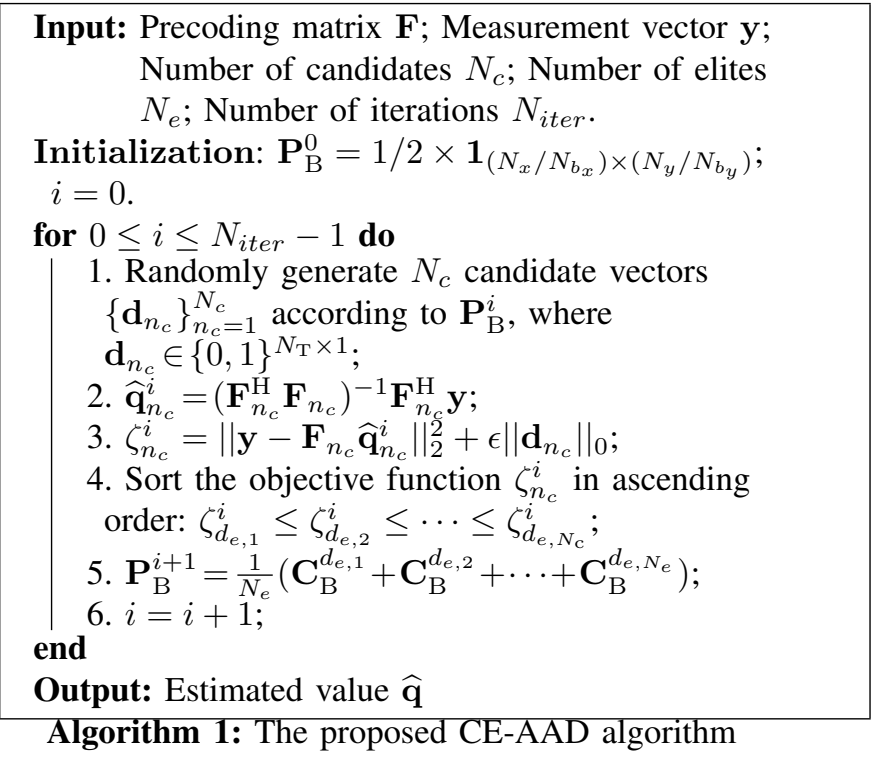

to compute $\widehat{\mathbf{q}}_{n_{c}}^{i} \in \mathbb{C}^{N_{f} \times 1}$, which is the estimate of the nonzero elements of $\mathbf{q}$ associated with the $n_{c}$-th candidate $\mathbf{d}_{n_{c}}$ in the $i$-th iteration. $N_{f}$ is the number of non-zero elements in candidate $\mathbf{d}_{n_{c}}$. We then define a submatrix $\mathbf{F}_{n_{c}} \in \mathbb{C}^{K \times N_{f}}$, which is composed of the column vectors of matrix $\mathbf{F}$ with indices belonging to $\mathcal{D}_{n_{c}}$. In Step 3 , we substitute $\widehat{\mathbf{q}}_{n_{c}}$ into the objective function $\zeta_{n_{c}}^{i}=\left\|\mathbf{y}-\mathbf{F}_{n_{c}} \widehat{\mathbf{q}}_{n_{c}}^{i}\right\|_{2}^{2}+\epsilon\left\|\mathbf{d}_{n_{c}}\right\|_{0}$, where $\left\|\mathbf{d}_{n_{c}}\right\|_{0}$ is the " $\ell_{0}$ " norm of vector $\mathbf{d}_{n_{c}}$, and $\epsilon$ is a superparameter [16], [17] which is proportional to the value of $\|\mathbf{y}\|_{2}^{2}$. In Step 4, we sort $\zeta_{n_{c}}^{i}$ in an ascending order and retain the first $N_{e}$ elements. Then, we select the corresponding $N_{e}$ candidates $\left\{\mathbf{C}_{\mathrm{B}}^{n_{c}}\right\}_{n_{c}=d_{e, 1}}^{d_{e, N_{e}}}$ as elites and record their indices as $\left\{d_{e, 1}, d_{e, 2}, \cdots, d_{e, N_{e}}\right\}$. In Step 5 , we update the probability matrix as $\mathbf{P}_{\mathrm{B}}^{i+1}=\frac{1}{N_{e}}\left(\mathbf{C}_{\mathrm{B}}^{d_{e, 1}}+\mathbf{C}_{\mathrm{B}}^{d_{e, 2}}+\cdots+\mathbf{C}_{\mathrm{B}}^{d_{e, N_{e}}}\right)$. We then repeat this procedure until the maximum number of iterations $N_{\text {iter }}$ is reached.

The traditional cross-entropy (CE) algorithm of [18], [20] (chapter 2) ignores the block-sparsity of the matrix $\mathbf{Q}$. In the contrast to the $\mathrm{CE}$ algorithm, the proposed CE-AAD algorithm generates $\mathbf{d}_{n_{c}}$ by exploiting the block-sparsity of $\mathbf{Q}$ in Step 1. The corresponding candidate matrix $\mathbf{C}_{n_{c}}$ exhibits a blockstructure. In this way, the CE-AAD algorithm achieves better performance of recovery and get faster convergence. Furthermore, when complete blockages occur, we have $a_{n}=0$. The proposed algorithm will be further simplified, since Step 2 and Step 3 can be combined. The objective function becomes $\zeta_{n_{c}}^{i}=\left\|\mathbf{y}-\mathbf{F} \mathbf{d}_{n_{c}}\right\|_{2}^{2}+\epsilon\left\|\mathbf{d}_{n_{c}}\right\|_{0}$. Hence it is not necessary to estimate the value of $\widehat{\mathbf{q}}_{n_{c}}^{i}$ by exploiting the LS algorithm. After $N_{i t e r}$ iterations, $\mathbf{d}_{d_{e, 1}}$ is the estimated value of $\mathbf{q}$.

The complexity of the proposed CE-AAD algorithm mainly comes from Steps 2 and 3. In Step 2, we calculate $\widehat{\mathbf{q}}_{n_{c}}^{i}=\left(\mathbf{F}_{n_{c}}^{\mathrm{H}} \mathbf{F}_{n_{c}}\right)^{-1} \mathbf{F}_{n_{c}}^{\mathrm{H}} \mathbf{y}$. The complexity of this part is $\mathcal{O}\left[N_{c}\left(N_{f} K N_{f}+K N_{f}+N_{f}^{2}+N_{f}^{3}\right)\right]$. In Step 3, we calculate $\zeta_{n_{c}}^{i}=\left\|\mathbf{y}-\mathbf{F}_{n_{c}} \widehat{\mathbf{q}}_{n_{c}}^{i}\right\|_{2}^{2}+\epsilon\left\|\mathbf{d}_{n_{c}}\right\|_{0}$. The complexity of this part is $\mathcal{O}\left[N_{c}\left(K+K N_{f}\right)\right]$. In summary, after $N_{\text {iter }}$ iterations, the total complexity of proposed CEAAD method is $\mathcal{O}\left[N_{i t e r} N_{c}\left(N_{f}^{3}+N_{f}^{2} K+2 K N_{f}\right)\right]$. Since

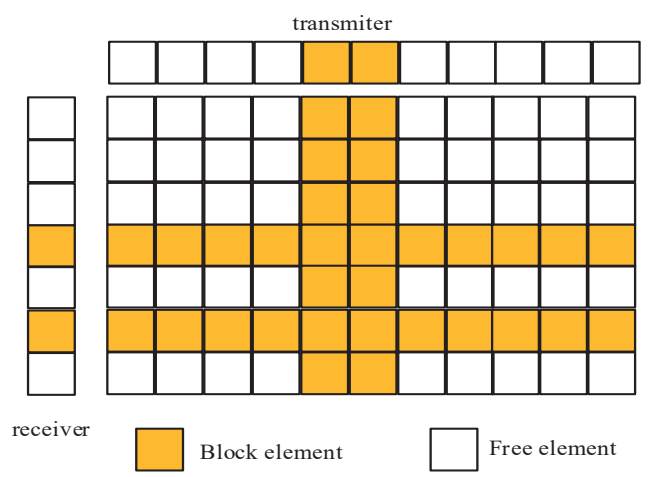

Fig. 2. An example of sparse structure of matrix $\mathbf{Q}$.

$N_{f} \leq K$ and the number of measurements $K$ is much smaller than $N_{t}$, we can conclude that the complexity of the proposed CE-AAD method is moderate, which is comparable to the method proposed in [12] (the complexity is $\left.\mathcal{O}\left[N_{\text {iter }}\left(N_{t}^{3}+N_{t}^{2} K+N_{t} K\right)\right]\right)$. When complete blockages occur, we have $a_{n}=0$. The complexity of proposed CE-AAD becomes $\mathcal{O}\left[N_{\text {iter }} N_{c}\left(K+K N_{f}\right)\right]$.

\section{JOINT CE-AAD}

In the previous section, we proposed the CE-AAD for the case where blockages only occur at the transmitter. In this section, we extend the proposed CE-AAD algorithm to the case where blockages simultaneously occur both at the transmitter and receiver.

We consider the uniform linear arrays (ULAs) of antennas at both the transmitter and receiver with $N_{\mathrm{t}}$ transmit antennas and $N_{\mathrm{r}}$ receive antennas ${ }^{1}$, respectively. Similar to (5), at the $k$-th measurement, we have

$$
y_{k}=r_{k}-g_{k}=\mathbf{w}_{k}^{\mathrm{H}} \underbrace{(\mathbf{H} \circ \mathbf{B}-\mathbf{H})}_{\mathbf{Q}} \mathbf{f}_{k}+\tilde{n}_{k},
$$

where $\mathbf{w}_{k} \in \mathbb{C}^{N_{\mathrm{r}} \times 1}$ denotes the combining vector at the receiver and $\mathbf{f}_{k} \in \mathbb{C}^{N_{\mathrm{t}} \times 1}$ represents the TPC vector at the transmitter. Furthermore, $\mathbf{B}=\mathbf{b}_{\mathrm{r}} \mathbf{b}_{\mathrm{t}}^{\mathrm{T}}$, where $\mathbf{b}_{\mathrm{r}} \in \mathbb{C}^{N_{\mathrm{r}} \times 1}$ and $\mathbf{b}_{\mathrm{t}} \in \mathbb{C}^{N_{\mathrm{t}} \times 1}$ denote the blockage coefficients at the receiver and transmitter, which have similar definitions as $\mathbf{b}$ in (3), while $\mathbf{H} \in \mathbb{C}^{N_{\mathrm{r}} \times N_{\mathrm{t}}}$ is the channel matrix of

$$
\mathbf{H}=\sum_{\ell=1}^{L} \beta_{\ell} \mathbf{a}_{\mathrm{r}}\left(\theta_{\ell}^{r}\right) \mathbf{a}_{\mathrm{t}}^{\mathrm{H}}\left(\theta_{\ell}^{t}\right)
$$

where $L$ is the number of propagation paths and $\beta_{l}$ is the complex gain of the $\ell$-th path. Furthermore, $\mathbf{a}_{\mathrm{r}}\left(\theta_{\ell}^{r}\right) \in$ $\mathbb{C}^{N_{\mathrm{r}} \times 1}$ and $\mathbf{a}_{\mathrm{t}}\left(\theta_{\ell}^{t}\right) \in \mathbb{C}^{N_{\mathrm{t}} \times 1}$ denote the antenna array responses at the receiver and transmitter, respectively. The steering vector $\mathbf{a}_{\mathrm{x}}\left(\theta_{\ell}^{\mathrm{x}}\right)$ can be expressed as $\left[1, e^{-j 2 \pi \frac{d}{\lambda} \sin \left(\theta_{\ell}^{\mathrm{x}}\right)}, \cdots, e^{-j 2 \pi \frac{\left(N_{\mathrm{x}}-1\right) d}{\lambda}} \sin \left(\theta_{\ell}^{\mathrm{x}}\right)\right]^{\mathrm{T}}$, where $\mathrm{x} \in\{\mathrm{t}, \mathrm{r}\}$ and $d$ is the antenna spacing. Vectorizing the matrix $\mathbf{Q} \in$

\footnotetext{
${ }^{1}$ The model in the ULA scenario can be easily extend to the UPA scenario.
} 


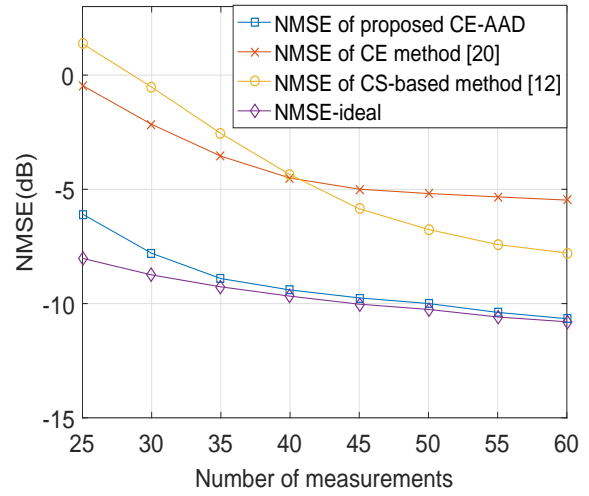

(a)

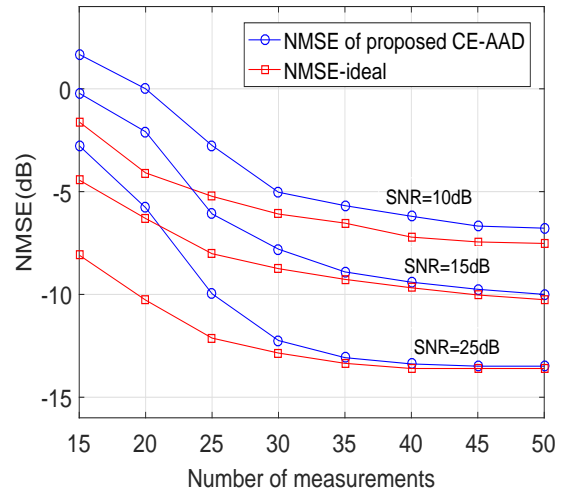

(b)

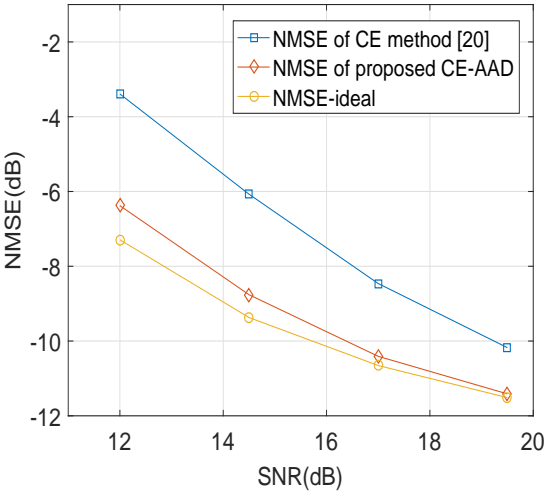

(c)

Fig. 3. NMSE performance comparison: (a) and (b) show the NMSE comparison when blockages occur at the transmitter. (c) shows the NMSE comparison when blockages occur at both the transmitter and receiver.

$\mathbb{C}^{N_{\mathrm{r}} \times N_{\mathrm{t}}},(8)$ can be rewritten as

$$
y_{k}=\underbrace{\left(\mathbf{f}_{k}^{\mathrm{T}} \otimes \mathbf{w}_{k}^{\mathrm{H}}\right)}_{\mathbf{u}_{k}} \underbrace{\operatorname{vec}(\mathbf{Q})}_{\mathbf{q}}+\widetilde{n}_{k},
$$

where the blockages occurring both at the transmitter and receiver may result in the matrix $\mathbf{Q}$ becoming of cross-shaped sparse structure, as shown in Fig. 2. After $K$ measurements, we have

$$
\mathbf{y}=\mathbf{U q}+\widetilde{\mathbf{n}},
$$

where $\mathbf{y}=\left[y_{1}, y_{2}, \cdots, y_{K}\right]^{\mathrm{T}} \in \mathbb{C}^{K \times 1}$ and $\mathbf{U}=$ $\left[\mathbf{u}_{1}^{\mathrm{T}}, \mathbf{u}_{2}^{\mathrm{T}}, \cdots, \mathbf{u}_{K}^{\mathrm{T}}\right]^{\mathrm{T}} \in \mathbb{C}^{K \times N_{r} N_{t}}$. The joint antenna array diagnosis becomes a sparse signal recovery problem.

Since the matrix $\mathbf{Q}$ has different sparse structures, the joint CE-AAD algorithm of this section is different from that in Section III. The main difference is reflected in Step 1 and Step 5. In Step 1, we generate the candidate vectors $\mathbf{d}_{\mathrm{t}}^{n_{c}} \in\{0,1\}^{N_{\mathrm{t}} \times 1}$ and $\mathbf{d}_{\mathrm{r}}^{n_{c}} \in\{0,1\}^{N_{\mathrm{r}} \times 1}$ according to the probability vectors $\mathbf{p}_{\mathrm{t}}^{i} \in \mathbb{R}^{N_{t} \times 1}$ and $\mathbf{p}_{\mathrm{r}}^{i} \in \mathbb{R}^{N_{r} \times 1}$. Then, we define the matrix $\mathbf{C}_{n_{c}} \in\{0,1\}^{N_{r} \times N_{t}}$, where the $(m, n)$ th element of matrix $\mathbf{C}_{n_{c}}$, denoted as $\mathbf{C}_{n_{c}}(m, n)$, indicates whether the $(m, n)$-th element of candidate matrix $\mathbf{Q}_{n_{c}}$ is a non-zero element. Thus, $\mathbf{C}_{n_{c}}(m, n)$ can be expressed as $\mathbf{C}_{n_{c}}(m, n)=\mathbf{d}_{\mathrm{r}}^{n_{c}}(m) \oplus \mathbf{d}_{\mathrm{t}}^{n_{c}}(n)$, where the operator $\oplus$ denotes the 'or' operation. In Step 5, we use $N_{e}$ elite vectors $\mathbf{d}_{\mathrm{t}}^{d_{e, k}}$ and $\mathbf{d}_{\mathrm{r}}^{d_{e, k}}\left(k=1,2, \cdots N_{e}\right)$ to update the probability vectors $\mathbf{p}_{\mathrm{t}}^{i}$ and $\mathbf{p}_{\mathrm{r}}^{i}$, which can be formulated as $\mathbf{p}_{t}^{i+1}=\frac{1}{N_{e}}\left(\mathbf{d}_{t}^{d_{e, 1}}+\mathbf{d}_{t}^{d_{e, 2}}+\cdots+\mathbf{d}_{t}^{d_{e, K}}\right)$ and $\mathbf{p}_{r}^{i+1}=\frac{1}{N_{e}}\left(\mathbf{d}_{r}^{d_{e, 1}}+\right.$ $\left.\mathbf{d}_{r}^{d_{e, 2}}+\cdots+\mathbf{d}_{r}^{d_{e, K}}\right)$, where $d_{e, k}$ has the same definition as in Section III. After obtaining the estimation of $\mathbf{q}$, we can identify the locations of blocked antennas and calculate the corresponding characteristic parameters.

\section{Simulation Results}

In this section, we characterize the performance of the proposed CE-AAD algorithm. We adopt the ray-based mmWave channel model of [19]. The system parameters used are as follows: $d=\frac{d_{x}}{\lambda}=\frac{d_{y}}{\lambda}=\frac{1}{2}, N_{c}=400, N_{e}=50, N_{i t e r}=20$, $\tau_{n} \sim U(0,1)$, and $\Psi_{n} \sim U(0,2 \pi)$. The number of dominant paths $L$ is $3, \beta_{\ell}$ follows the Gaussian distribution with zero mean and unit variance, while $\theta_{\ell}, \varphi_{\ell}, \theta_{\ell}^{r}, \theta_{\ell}^{t}$ are randomly chosen from $[-\pi / 2, \pi / 2]$. We assume that the group blockage probability is $p_{g}=0.1$ (the size of blockage group is $2 \times 2$ ) in Fig. 3 (a) and Fig. 3 (b) and the blockage probability of the antennas is $p_{b}=0.1$ in Fig. 3 (c). We simulate the normalized mean square error (NMSE) of $\mathbf{b}$ and $\mathbf{B}$ estimated both by the proposed CE-AAD method and by a pair of two traditional AAD methods [12], [20].

In Fig. 3 (a) and (b), we consider a single free antenna at the receiver and a UPA having $10 \times 10$ antennas at the transmitter. Fig. 3 (a) shows the NMSE comparison of different algorithms vs. the number of measurements when the signalto-noise ratio (SNR) $\frac{\|\mathbf{F q}\|_{2}^{2}}{K \delta^{2}}$ is $15 \mathrm{~dB}$. NMSE-ideal represents the performance upper-bound, which is achived when the exact locations of blocked antennas are known. We observe that the proposed algorithm outperforms other algorithms. In Fig. 3 (b), we compare the NMSE performance of the proposed algorithm and NMSE-ideal at different SNRs. We observe that the proposed algorithm achieves near-ideal performance using only a few measurements.

In Fig. 3 (c), we consider the case where blockages occur at both the transmitter and receiver, which rely on the ULAs of antennas having 10 elements. Fig. 3 (c) shows the NMSE performance comparison vs. SNRs when the number of measurements is 50 . We observe that the proposed joint CE-AAD algorithm has a good performance.

In Fig. 4, we consider a single free antenna at the receiver and a UPA having $12 \times 12$ antennas at the transmitter. The number of iterations $N_{\text {iter }}$ is 25 . We observed that the NMSE performance of the proposed algorithm is improved as $N_{c}$ increases and it is not sensitive to the parameter $N_{e}$. When $N_{c}$ is higher than 190, the NMSE performance becomes stable.

\section{CONCLUSIONS}

In this paper, we studied the AAD problem of mmWave MIMO systems. By exploiting the correlations between adjacent blocked antennas, we proposed the CE-AAD algorithm for identifying the locations and characteristic parameters of 


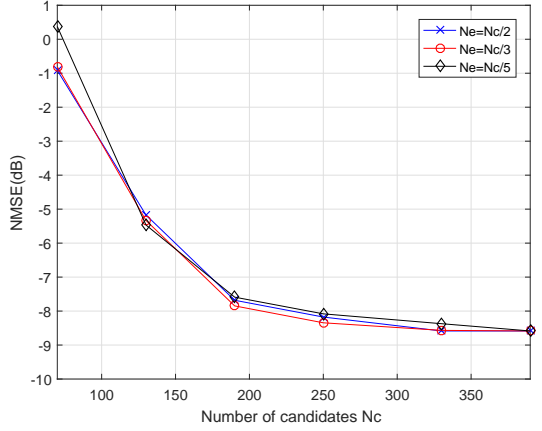

Fig. 4. The NMSE performance against the $N_{c}$ and $N_{e}$.

blocked antennas when blockages occurs at the transmitter. Then, we extended the proposed CE-AAD algorithm to the case, where blockages occur both at the transmitter and receiver simultaneously. Our simulation results verify that the proposed method achieves near-optimal performance, approaching that of the genie solution having explicit knowledge of the blocked antenna indices.

\section{REFERENCES}

[1] X. Gao, L. Dai, and A. M. Sayeed, "Low RF-complexity technologies to enable millimeter-wave MIMO with large antenna array for 5G wireless communications," IEEE Commun. Mag., vol. 56, no. 4, pp. 211-217, Apr. 2018.

[2] R. Rajashekar and L. Hanzo, "Hybrid beamforming in mm-Wave MIMO systems having a finite input alphabet," IEEE Trans. Commun., vol. 64 , no. 8, pp. 3337-3349, Aug. 2016.

[3] K. Erdoğan and H. A. Ilgin, "Analysis of atmospheric effects on millimeter wave frequency bands for fifth generation mobile networks," in Proc. ISMSIT, Oct. 2018, pp. 1-4.

[4] O. Harel, N. David, P. Alpert, and H. Messer, "The potential of microwave communication networks to detect dew-experimental study," IEEE J-STARS., vol. 8, no. 9, pp. 4396-4404, Sep. 2015.

[5] A. Arage, W. M. Steffens, and G. Kuehnle, "Effects of water and ice layer on automotive radar," in Proc. German Microw, Mar. 2006, pp. $1-5$.

[6] P. Zhang, L. Gan, C. Ling, and S. Sun, "Atomic norm denoisingbased joint channel estimation and faulty antenna detection for massive MIMO," IEEE Trans. Veh Technol., vol. 67, no. 2, pp. 1389-1403, Feb. 2018.

[7] O. M. Bucci, A. Capozzoli, and G. D'Elia, "Diagnosis of array faults from far-field amplitude-only data," IEEE Tran. Antennas Propag., vol. 48, no. 5, pp. 647-652, May 2000.

[8] O. M. Bucci, M. D. Migliore, G. Panariello, and P. Sgambato, "Accurate diagnosis of conformal arrays from near-field data using the matrix method," IEEE Tran. Antennas Propag., vol. 53, no. 3, pp. 1114-1120, Mar. 2005.

[9] M. D. Migliore, "A compressed sensing approach for array diagnosis from a small set of near-field measurements," IEEE Tran. Antennas Propag., vol. 59, no. 6, pp. 2127-2133, Jun. 2011.

[10] Z. Liu and Y. Zhou, "A unified framework and sparse bayesian perspective for direction-of-arrival estimation in the presence of array imperfections," IEEE Trans. Signal Process., vol. 61, no. 15, pp. 37863798, Aug. 2013.

[11] C. Xiong, G. Xiao, Y. Hou, and M. Hameed, "A compressed sensingbased element failure diagnosis method for phased array antenna during beam steering," Antennas Wireless Propagat. Lett., vol. 18, no. 9, pp. 1756-1760, Sep. 2019.

[12] M. E. Eltayeb, T. Y. Al-Naffouri, and R. W. Heath, "Compressive sensing for millimeter wave antenna array diagnosis," IEEE Trans. Commun., vol. 66, no. 6, pp. 2708-2721, Jun. 2018.

[13] W. Shen, L. Dai, B. Shim, Z. Wang, and R. W. Heath, "Channel feedback based on AoD-adaptive subspace codebook in FDD massive MIMO systems," IEEE Trans. Commun., vol. PP, no. 99, pp. 1-1, Jun. 2018.
[14] M. E. Eltayeb, "Relay-aided channel estimation for mmWave systems with imperfect antenna arrays," in Proc. IEEE ICC, May 2019, pp. 1-5.

[15] S. M. Razavi and T. Ratnarajah, "Adaptive LS- and MMSE-based beamformer design for multiuser MIMO interference channels," IEEE Trans. Veh Technol., vol. 65, no. 1, pp. 132-144, Jan. 2016.

[16] F. Wu, K. Yang, and R. Duan, "Compressed sensing of underwater acoustic signals via structured approximation $l_{0}$ norm," IEEE Trans. Veh Technol., vol. 67, no. 9, pp. 8504-8513, Sep. 2018.

[17] M. Borgerding, P. Schniter, and S. Rangan, "AMP-inspired deep networks for sparse linear inverse problems," IEEE Trans. Signal Process., vol. 65, no. 16, pp. 4293-4308, Aug. 2017.

[18] X. Gao, L. Dai, Y. Sun, S. Han, and I. Chih-Lin, "Machine learning inspired energy-efficient hybrid precoding for mmWave massive MIMO systems," in Proc. IEEE ICC, May 2017, pp. 1-6.

[19] R. W. Heath, N. Gonzáez-Prelcic, S. Rangan, W. Roh, and A. M. Sayeed, "An overview of signal processing techniques for millimeter wave MIMO systems," IEEE J. Sel. Top. Signal Process., vol. 10, no. 3, pp. 436-453, Apr. 2016.

[20] R. Rubinstein and D. Kroese, "The cross-entropy method: A unified approach to combinatorial optimization, Monte-Carlo simulation and machine learning," Springer Science\&Business Media, 2013. 